\title{
Precisely delivered nanomechanical forces induce blebbing in undifferentiated mouse embryonic stem cells
}

This article was published in the following Dove Press journal:

Cell Health and Cytoskeleton

25 January 2011

Number of times this article has been viewed

\section{Alexandra L Hemsley' \\ Diana Hernandez' \\ Christopher Mason' \\ Andrew E Pelling 2,3 \\ Farlan S Veraitch'}

'Advanced Centre for Biochemical Engineering, ${ }^{2}$ The London Centre for Nanotechnology, Centre for Nanomedicine, University College London, London, UK; ${ }^{3}$ Department of Physics, University of Ottawa,

Ottawa, ON, Canada
Correspondence: Andrew E Pelling Department of Physics, University of Ottawa, MacDonald Hall, I50 Louis Pasteur, Ottawa, ON KI N 6N5, Canada $\mathrm{Tel}+\mathrm{I} 6 \mid 35625800$ ext 6965

$\mathrm{Fax}+16135625190$

Email a@pellinglab.net

Correspondence: Farlan S Veraitch Advanced Centre for Biochemical Engineering, University College London, Torrington Place, London WCIE 7JE, UK

Tel +442076792648

Fax +44 2072090703

Email f.veraitch@ucl.ac.uk

\begin{abstract}
The aim of this study was to probe the morphological response of single mouse embryonic stem cells (mESC) to precisely delivered nanomechanical forces. Plating mESC as single cells gave rise to either round compact or flattened fibroblastic morphologies. The expression of OCT4 and Nanog was reduced in flattened cells, indicating that this population had begun to differentiate. Upon application of $>5 \mathrm{nN}$ of force, using atomic force microscopy and simultaneous laser scanning confocal microscopy, round cells, but not flattened cells, were capable of forming mechanically induced blebs (miBlebs). Flattened cells appeared to have a more highly developed cytoskeleton than undifferentiated stem cells as characterized by the distribution of phospho-ezrin-radixin-moesin (pERM). Higher levels of pERM and an inability to form miBlebs in flattened cells imply that the earliest stages of embryonic stem cell differentiation are associated with the development of stronger mechanical links between the plasma membrane and the cytoskeleton.
\end{abstract}

Keywords: blebbing, embryonic stem cells, atomic force microscopy, cytoskeleton development, differentiation

\section{Introduction}

Embryonic stem cells (ESC) are derived from the inner cell mass at the blastocyst stage of early embryos. They can be maintained indefinitely in an undifferentiated state, while retaining their capacity to differentiate into lineages from all three germ layers, making them ideal candidates for regenerative medicine, drug discovery, and drug development. Every aspect of the microenvironment must be tightly controlled to enable robust, reproducible, and high-yield production of ESC-derived cellular preparations. ${ }^{1}$ Recently, it has become evident that the mechanical microenvironment of a cell, in concert with morphology, biochemical signals, and cellular responses, can dictate diverse processes such as differentiation, mitosis, and malignant transformation. ${ }^{2-6}$ The cellular microenvironment includes the culture surface, the extracellular matrix (ECM), substrate composition and stiffness, the concentration of dissolved gases, signaling molecules, metabolites, cell-to-cell contact, and extracellular physical forces. A number of studies have revealed that ESC proliferation and ESC differentiation can be influenced by exogenous forces. ${ }^{7-11}$ In addition, the differentiation of mesenchymal stem cells was found to be sensitive to the stiffness of the underlying substrata. ${ }^{2}$ To further investigate the response of $\mathrm{mESC}$ to specific forces, we employed atomic force microscopy (AFM) and simultaneous laser scanning confocal microscopy (LSCM), ${ }^{12,13}$ thereby enabling the application of local, temporally and spatially controlled forces without inducing massive deformations. 
Here, we show that two subpopulations exist within single undifferentiated mESC. These subpopulations are divided by morphological (round and flattened) and biochemical lines. We observed that round cells displayed significant bleb formation and retraction, while cells with flattened morphologies displayed none. Biochemical assays revealed that round cells had higher expression levels of the pluripotent markers OCT4 and Nanog than flattened morphologies. Interestingly, precisely delivered extracellular forces (via AFM) resulted in the formation of blebs on the cell membrane. Blebbing is a process in which protrusions of the plasma membrane form when the plasma membrane separates from the cell cortex. Blebbing is believed to have a role in the production of plasma membrane proteins prior to cell division, and may also help to generate new plasma membrane proteins prior to mitosis and assist in cell movement. ${ }^{14-16}$ Mechanically induced blebs (miBlebs) only occurred in mESC with round morphologies. Flattened cells did not display miBlebs upon application of force. We found that expression of active phospho-ezrin-radixin-moesin (pERM) in cells with flattened morphologies was significantly higher than in the round cells. pERM proteins are well known to link the plasma membrane to the actin cytoskeleton and provide a direct link between the external and internal mechanical microenvironments of the cell. ${ }^{5}$ We suggest that increased activity of these proteins may occur very early during mESC differentiation.

\section{Materials and methods \\ Cell culture and transfections}

OCT4-GiP is a mESC line previously transfected with the Oct4-GFPirespac transgene, ${ }^{17}$ which expresses GFP under direction of regulatory elements of the mouse OCT4 gene. OCT4-GiP and the parental line E14tg2a (kindly donated by Stem Cell Sciences Ltd, Cambridge, UK) cells were routinely passaged following a previously published protocol. ${ }^{9}$ Briefly, cells were cultured in Glasgow Minimum Essential Media (Sigma Aldrich, Dorset, UK), 10\% fetal calf serum (FCS; Invitrogen, Paisley, UK), 1\% MEM nonessential amino acids (Gibco, Paisley, UK), 1\% 2- $\beta$ mercaptoethanol (Gibco), and $1 \%$ glutamate-pyruvate (Invitrogen, Carlsbad, CA), with or without leukemia inhibitory factor (LIF; Millipore, Watford, UK) $(1 \mu \mathrm{L} / \mathrm{mL})$. Cells were routinely passaged every 2 days in Iwaki flasks (SLS, Nottingham, UK) coated with $0.1 \%$ gelatine (Sigma). AFM experiments were conducted with OCT4-GiP cells and repeated with E14tg2a cells. Unless otherwise stated, cells were plated in gelatinized $60-\mathrm{mm}$ Iwaki Petri dishes and cultured overnight at a low seeding density $\left(5000\right.$ cells $\left./ \mathrm{cm}^{2}\right)$ to yield a significant number of single cells for analysis. The plasma membrane of E14tg2a cells was imaged using a GFP-tagged PH domain of phospholipase C- $\delta$ as described previously (a kind gift of Dr. Guillaume T Charras, London Centre for Nanotechnology, London, UK). ${ }^{14}$ Transient transfections were effected using Lipofectamine $^{\mathrm{TM}} 2000$ (Invitrogen) and $1 \mu \mathrm{g}$ of DNA according to the manufacturer's protocol. Transfected cells were plated onto gelatine-coated $60-\mathrm{mm}$ glass-bottomed dishes (World Precision Instruments, England, UK) and imaged the next day.

\section{Immunofluorescent staining and imaging}

All cells were fixed and stained using standard immunohistochemical methods and as outlined in detail in the supplementary methods, having been left to attach overnight for $\sim 16 \mathrm{~h}$. Briefly, all cells were fixed with 3.5\% paraformaldehyde (Sigma) and 2\% sucrose (Sigma) and then permeabilized with $0.1 \%$ Triton X-100 (Sigma). Finally, cells were blocked in wash buffer ( $5 \%$ FCS in phosphate buffered saline [PBS] for $20 \mathrm{~min}$ ). Actin was stained with Phalloidin conjugated to Alexa Fluor 546 or 488 (Invitrogen) in E14tg2a cells. E14tg2a cells were incubated with mouse monoclonal $\alpha$-tubulin (Sigma) or rabbit polyclonal pERM antibodies overnight in the fridge (Cell Signalling Technologies, Danvers, MA, USA), a kind gift of Dr. Buzz Baum (MRC Laboratory for Molecular Cell Biology, London, UK). Rabbit anti-mouse or goat anti-rabbit secondary antibodies conjugated to Alexa Fluor 488 and 647 (Invitrogen), respectively, were applied to the cells followed by wash buffer. Finally, nuclei were stained with 4',6-diamidino-2-phenylindole (DAPI; Invitrogen). To stain for Nanog or OCT4, cells were incubated with rabbit polyclonal anti-Nanog (Abcam, Cambridge, UK) (OCT4-GiP cells) or mouse monoclonal OCT4 (Santa Cruz Biotechnology, Santa Cruz, CA, USA) (E14tg2a cells). Cells were then incubated with anti-rabbit secondary antibodies conjugated to Alexa Fluor 647 or anti-mouse Alexa Fluor 555 (Abcam), respectively. The cells were then washed with PBS and stained with DAPI as above. Finally, cells were rinsed and imaged in PBS.

Image J (see http://rsb.info.nih.gov/ij) was used to quantify the total fluorescence value per cell. Values were normalized by dividing the fluorescence value by the background value using the same region of interest. The morphology of the cells was identified by cross-referencing the fluorescent images with the corresponding phase-contrast image. Images of the cytoskeleton and pERM were acquired with an FV1000 IX81 laser scanning confocal microscope 
(Olympus, Southend-on-Sea, UK). Images of OCT4-GFP, Nanog, and OCT4 were obtained using a Nikon Eclipse TE2000-U fluorescent microscope (Nikon, Surrey, UK). A BioVision Annexin V-FITC Apoptosis Detection Kit (BioVision, Mountain View, CA) was used to confirm E14tg2a cells were not apoptotic. The staining kit includes propidium iodine (PI), which was used to assess cell membrane integrity. The manufacturer's protocol was followed and cells were subsequently imaged. As a positive control, the apoptosis inducer staurosporine ( $4 \mu \mathrm{M}$; Sigma) was added $16 \mathrm{~h}$ prior to analysis. Using Image $\mathrm{J}$, the area of OCT4-GiP cells and corresponding nuclei were determined manually $(\mathrm{n}=50)$ and used to calculate the nuclear:cytoplasm area ratio.

\section{AFM}

A NanoWizard I AFM (JPK Instruments, Berlin, Germany) mounted on an inverted phase-contrast and fluorescence microscope (IX71; Olympus) or an inverted LSCM (IX81 FV1000; Olympus) was used for all experiments. MSCTAUHW (Veeco, Cambridge, UK) $\mathrm{SiN}_{4}$ cantilevers with pyramidal tips and experimentally determined (via the thermal fluctuation method) spring constants of $k=0.02 \pm 0.01 \mathrm{~N} / \mathrm{m}$ were used. HEPES buffer (10 $\mathrm{mM}$ final concentration) was added to the culture media $1 \mathrm{~h}$ prior to experiments. Resistive heating of the microscope stage maintained a temperature of $37^{\circ} \mathrm{C}$. For all experiments, a selected cell was aligned 1-5 $\mu \mathrm{m}$ below the AFM tip. The AFM tip was lowered at a speed of $5 \mu \mathrm{m} / \mathrm{sec}$ directly onto the nucleus of flattened cells or over the center of round cells, and a constant force was applied to the cell. Phase-contrast videos were recorded at 1 frame/sec with a deep-cooled CCD camera (Hamamatsu, Welwyn Garden City, UK).

\section{Statistical analysis}

Unless otherwise stated, all cell culture experiments were conducted in triplicate. Significant differences between data points were calculated using a two-tailed, paired Student's $t$-test. Differences in data sets were deemed to be significantly different when $P \leq 0.05$. Error bars represent standard deviation. The $\mathrm{n}$ values have been given in the appropriate figure legends.

\section{Results}

\section{Biochemical analysis of morphologically distinct subpopulations of single cells}

Prior to AFM experiments, we characterized the effect of low seeding density on morphology, pluripotency, and checked for the induction of apoptosis. OCT4-GiP and E14tg2a
mESC were seeded at 5000 cells $/ \mathrm{cm}^{2}$ to yield single cells for single cell AFM analysis. After an overnight culture, we observed the formation of two clear morphologically distinct populations. Round cells were defined as circular, compact cells with very clear cell borders. Flat cells appeared more spread and had jagged cell borders (Figure 1A, B). The round and flattened morphological subpopulations were both well attached to the culture surface and detached at the same rate when trypsinized. Phase-contrast images of the cell borders of flat cells were less clear than round cells. Using these morphological criteria, we found that $\sim 47 \%$ $( \pm 14 \%)$ of the single cells were flat cells while $36 \%( \pm 12 \%)$ of the population were round. A proportion of the round cells were spontaneously forming and retracting small blebs (Figure S1). No spontaneous blebbing was observed in the subpopulation of flattened cells. In order to confirm that round cells were not apoptotic, we stained cells with Annexin V-FITC to screen for apoptotic cells. As a positive control, the well-known protein kinase inhibitor, staurosporine, was used to induce apoptosis. ${ }^{18}$ Neither the round or flattened cellular morphologies were apoptotic (Figure 1A, B), whereas all cells cultured in the presence of staurosporine were positive for Annexin V (Figure 1C).

Having established that low seeding densities had not induced apoptosis, we characterized the expression of pluripotency markers in the two morphologically distinct subpopulations of single cells. In OCT4-GiP cells, we found that GFP expression was significantly higher in round cells $(P \leq 0.05)$ (Figure 2A). This suggests that flattened cells are in the early stages of differentiation resulting in a decrease, but not a complete loss, of OCT4 expression. In order to confirm this, we also tested for Nanog and OCT4 expression levels in wild-type E14tg2a cells using immunofluorescence microscopy. These data (Figure 2B, C) revealed lower levels of both Nanog $(P \leq 0.05)$ and OCT4 $(P \leq 0.05)$ in flattened cells as compared to round cells supporting the notion that flattened cells were spontaneously differentiating. Ideally, differences in pluripotency marker expression would be verified with techniques such as RT-PCR and Western blotting. However, flattened and round cells could not be separated by selective detachment or cell sorting once they have been detached from the culture surface. This technical limitation also meant that flat and round cells could not be recultured separately. Therefore, we relied upon in situ immunocytochemistry analysis for assaying pluripotency marker expression. It has been observed that pluripotent ESC typically have larger nuclei than many other cell types and therefore have a larger nucleus:cytoplasm ratio than differentiated cells. ${ }^{19}$ 

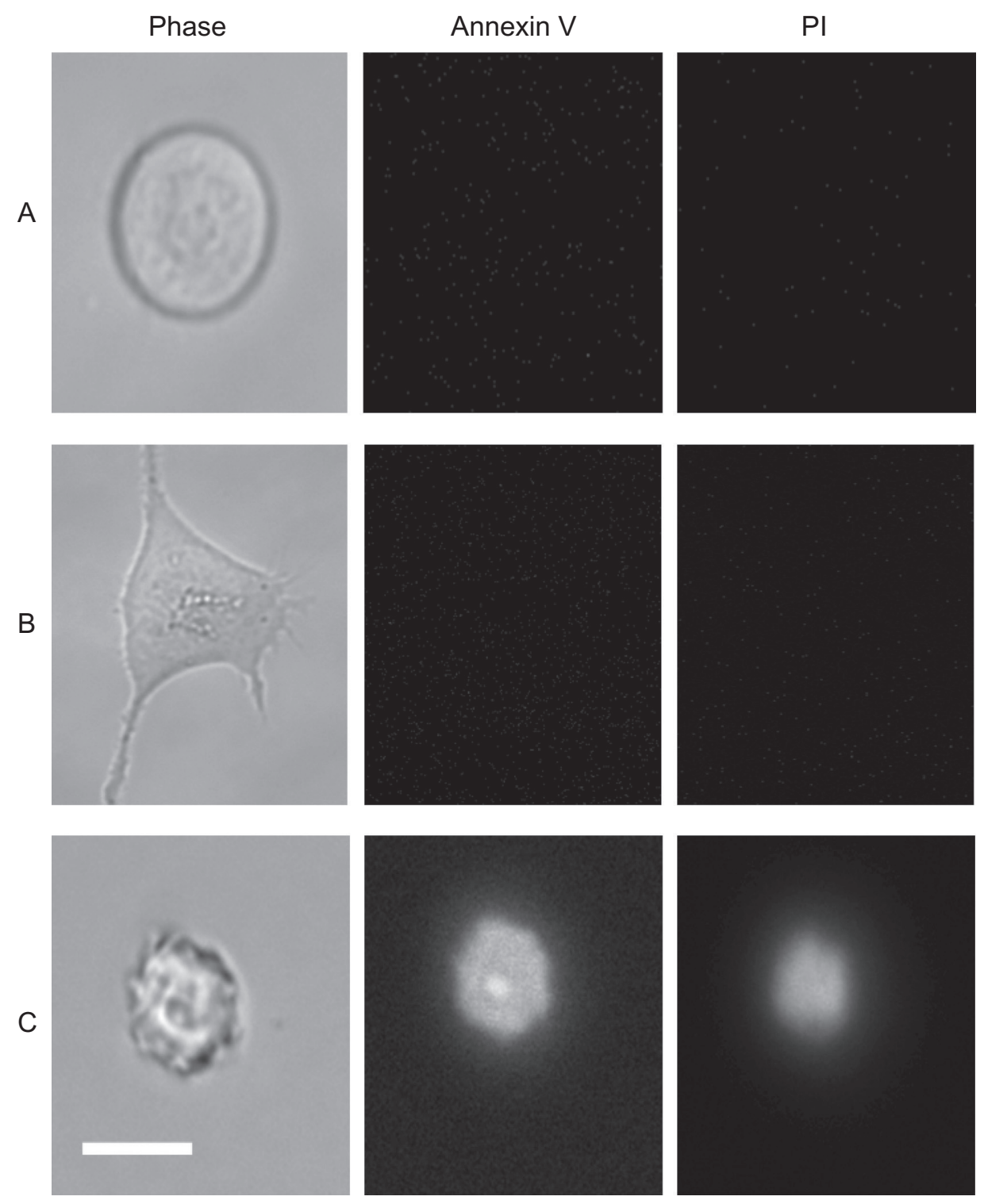

Figure I Culturing EI4tg2a mESC overnight at a low cell density resulted in the formation of two distinct morphological subpopulations. A) Round cells were compact while B) flattened cells had spread out and displayed a fibroblast-like morphology. To confirm cells were not displaying apoptotic protrusions or undergoing apoptosis due to the low seeding density, the cells were assayed for apoptosis. The images above demonstrate that neither single round nor flattened cells are positive for Annexin $V$ or $\mathrm{Pl}$ at a low seeding density. C) Positive controls, which were positive for Annexin $\mathrm{V}$ and $\mathrm{PI}$, had been treated with $4 \mu \mathrm{M}$ staurosporine for $16 \mathrm{~h}$ prior to the apoptosis assay (scale bar $=8 \mu \mathrm{m}$ for all images).

We first measured the nuclear and cytoplasmic areas (Figure S2) which revealed that round cells have larger nuclei than flat cells. Consistent with the results for OCT4-GFP, OCT4, and Nanog fluorescent intensities, the nuclear:cytoplasm ratio was significantly higher in round versus flattened single cells $(P \leq 0.05)$ confirming again that single flattened cells have begun to differentiate (Figure 2D). In order to further investigate whether spontaneous blebbing was a dynamic morphological trait associated with the pluripotent state, we induced differentiation by culturing cells for $0,2,4,6$, and 8 days in the absence of LIF. ${ }^{20}$ As illustrated in Figure S1, the percentage of single cells spontaneously blebbing decreased significantly after inducing differentiation upon the removal of LIF. The decrease in the number of blebbing cells on day 0 compared with day 8 (after LIF removal) was found to be statistically significant $(P \leq 0.05)$.

\section{Mechanically induced blebbing in round cells}

The two morphological subpopulations of single mESC provide a model platform to investigate the response of these cells to controlled mechanical forces. Therefore, we employed AFM to apply controlled forces $(1,5$, and $10 \mathrm{nN})$ to both round and flattened cells. 
A

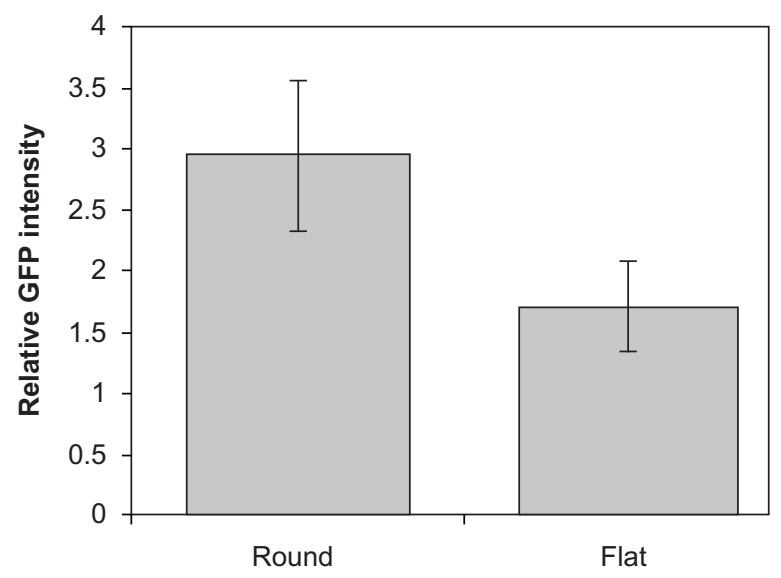

C

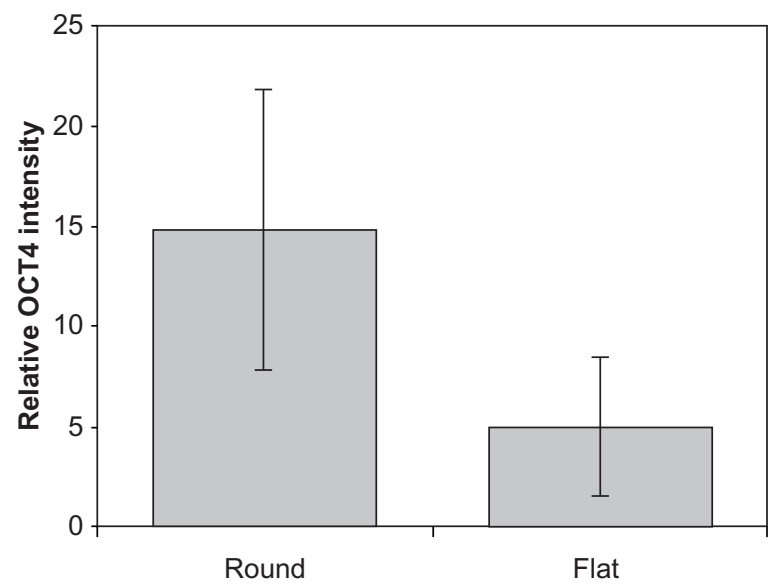

B

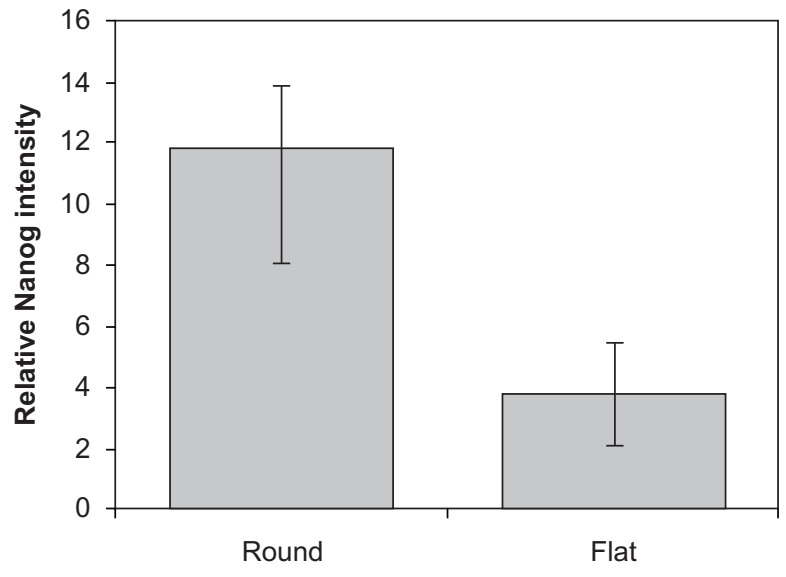

D

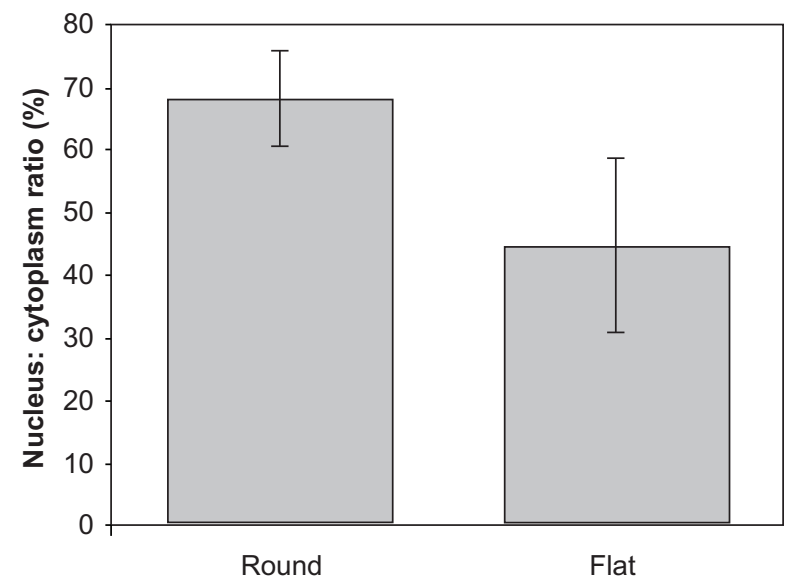

Figure 2 Analysis of pluripotency marker expression of subpopulations of single cells using immunofluorescence microscopy. We imaged A) OCT4-GFP intensity as well as the B) intensity of fluorescently stained Nanog and C) OCT4 transcription factors. Twenty-five cells of each morphological subpopulation were analyzed for OCT4 (EI4tg2a cells), Nanog (OCT4-GiP cells), and GFP (OCT4-GiP cells) intensity measurements in triplicate cultures $(n=3)$. In each case, round cells had a significantly higher expression of these pluripotency factors. D) The decrease in expression suggests that flattened cells are in the early stages of differentiation, supported by the qualitative observation that the nucleus:cytoplasm ratio (OCT4-GiP cells) is higher in round cells.

Application of force to $\mathrm{mESC}$ resulted in a downward compression of the cell as would be expected. A constant force was applied to each cell for $30 \mathrm{sec}$ followed by retraction and removal of the AFM tip. While this took place, time lapse imaging ( 1 frame/sec) was carried out for several minutes. On application of 5 or $10 \mathrm{nN}$ of force to round cells, we immediately observed the formation of 1-3 mechanically induced miBlebs, which did not occur for applied forces of $1 \mathrm{nN}$ (Figure 3A and Figure S3). miBlebs were typically $>2$ times larger than spontaneously formed blebs (Figure 3B) and were observed to appear 1-2 sec after application of force in the majority of cells tested ( $80 \%)$. Typically, miBlebs expanded to an equilibrium size in 10-30 sec, and after the removal of force, miBlebs retracted and disappeared within another $30 \mathrm{sec}$. These bleb dynamics correspond well to previous observations of bleb dynamics. ${ }^{14}$ Furthermore, the application of force to round cells, which already possessed spontaneously formed blebs, resulted in the formation of new miBlebs rather than the expansion of an existing bleb (Figure 3B). Importantly, the application of 1, 5, and $10 \mathrm{nN}$ forces to flattened cells in a variety of locations resulted in no miBleb (or spontaneous bleb) formation (Figure 3C). In addition, cells that had been allowed to differentiate in the absence of LIF for 8 days did not form miBlebs regardless of morphology (results not shown). To observe the dynamics of the membrane more directly, we transfected the cells with DNA encoding a GFP-tagged PH domain of phospholipase $\mathrm{C}-\delta$ (GFP-PLC $\delta$ ). Utilizing simultaneous AFM and LSCM, cells were imaged prior to the application of force and after $30 \mathrm{sec}$, at which point images of a single confocal plane (containing a miBleb) were imaged at 1 -sec intervals for the next $2 \mathrm{~min}$ (Figure 3D). This data clearly reveals the 
$t=-10 \sec$

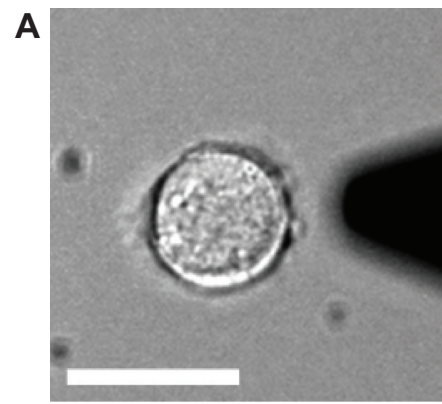

B

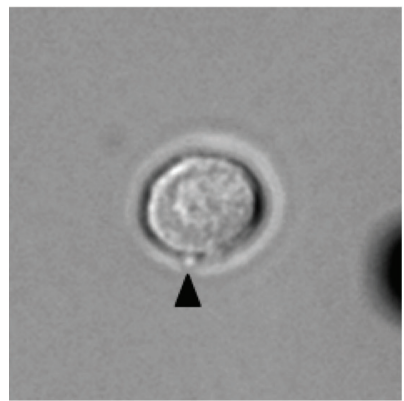

C

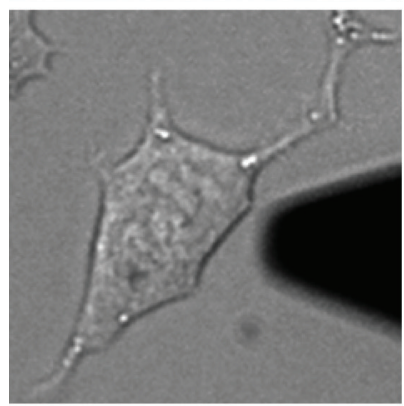

$\mathrm{t}=\mathbf{0} \sec$
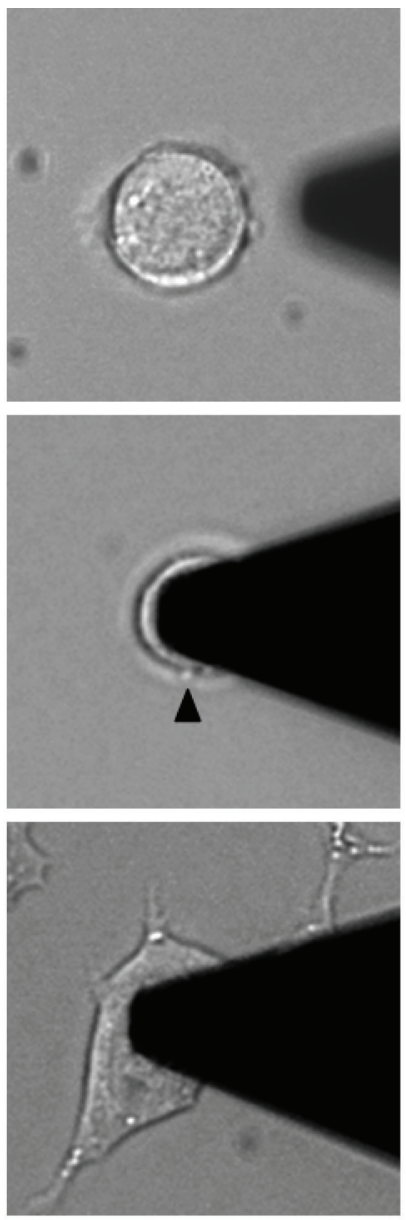

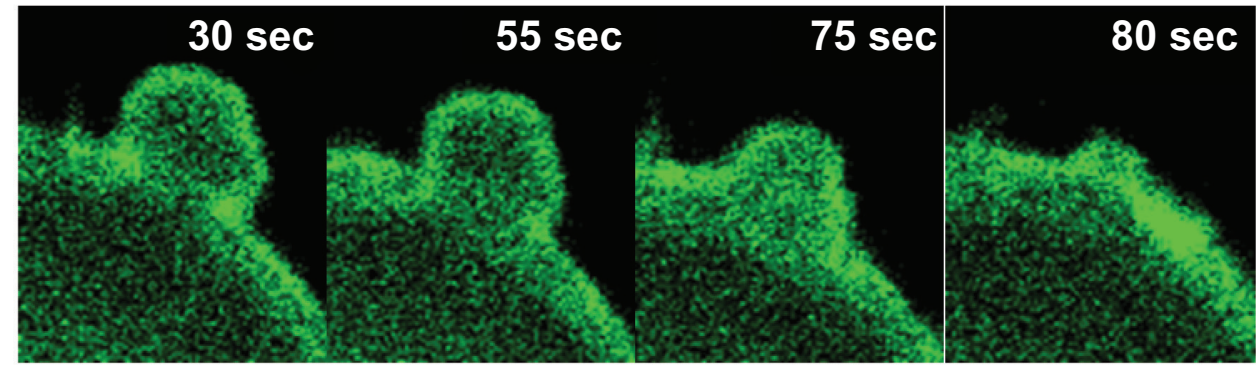

$t=30 \mathrm{sec}$
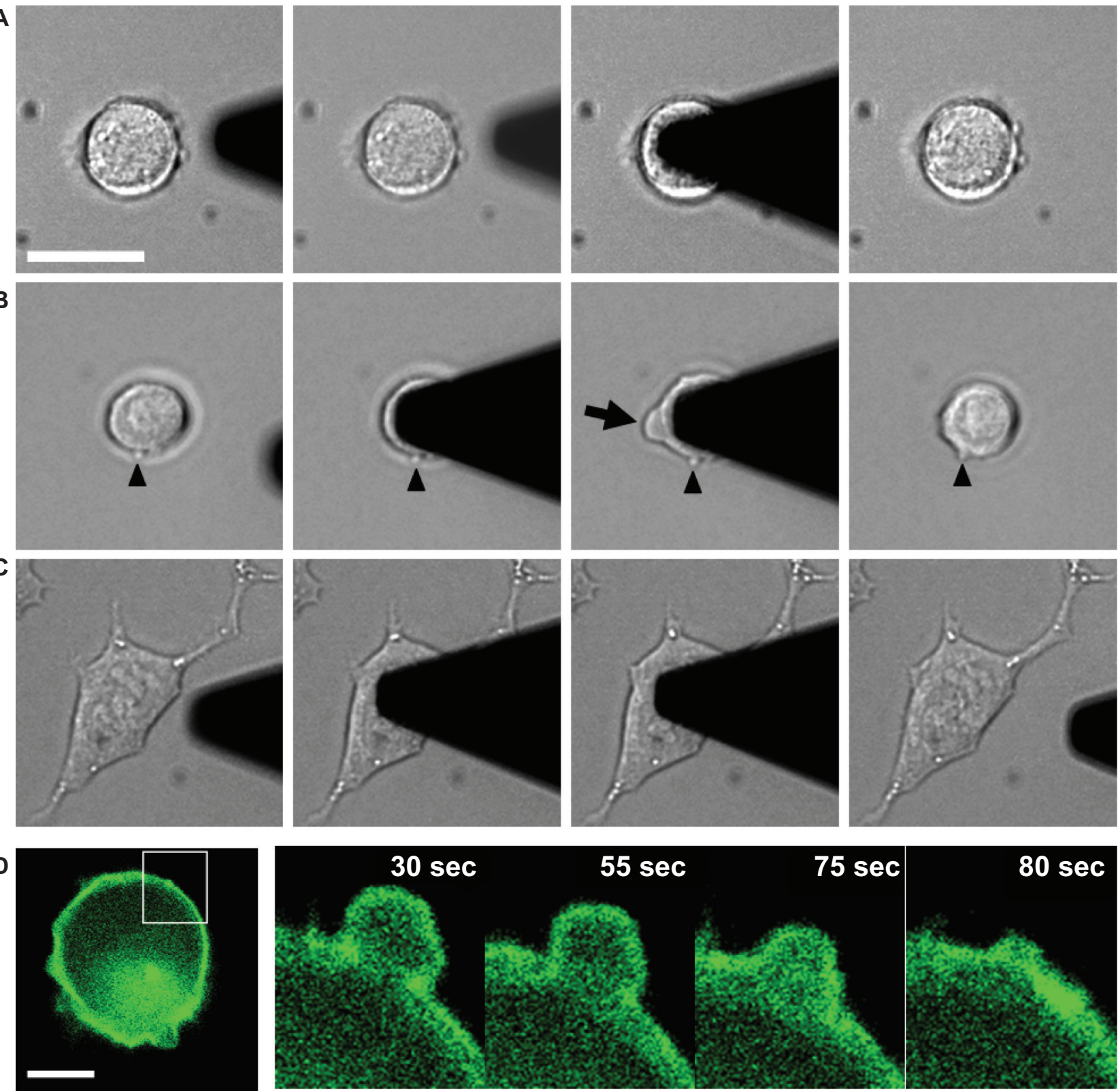

Figure 3 AFM and LSCM were utilized to apply well-defined forces to single OCT4-GiP mESC (scale bar $=10 \mu \mathrm{m}$, applies A-C) and monitor their reaction. A) Forces of I $\mathrm{nN}$ did not result in miBlebs $(\mathrm{n}=10$ cells). B) However, force of $5 \mathrm{nN}(\mathrm{n}=10, \mathrm{S3})$ and $10 \mathrm{nN}(\mathrm{n}=25)$ resulted in the formation of miBlebs (arrow) in the majority of cells studied ( $80 \%)$. Small spontaneous blebs which were present prior to the application of force (triangle) did not grow in size during force application and remained after retraction of the miBleb. C) Flattened cells did not form miBlebs ( $10 \mathrm{nN}$ in these images). D) Expression of GFP-PLC $\delta$ in the plasma membrane allowed us to directly image the miBleb formation and retraction (scale bar $=2 \mu \mathrm{m}$ ). An image stack of the cell was collected prior to the application of force and after 30 sec to determine the location of the miBleb. The z-plane which contained the miBleb was then imaged every second after removal of force. miBleb retraction and cell relaxation is clearly observable after removal of force.

retraction of the miBleb and resulting relaxation of the cell membrane after application of force.

The cytoskeleton and ERM proteins are central elements which define and regulate cell morphology and mechanotransduction. ${ }^{4,5}$ In order to gain insight into the ability for round cells to form miBlebs, we imaged the actin and microtubule cytoskeleton as well as the expression of activated pERM proteins. In both round and flattened mESC, we observed cortical actin and an absence of large F-actin bundles. The actin network was also clearly found around spontaneous blebs under the membrane (Figure 4A, B). The microtubule cytoskeleton was found to be well defined and filamentous in round and flattened cells. In flattened cells, intact microtubules were found extending 

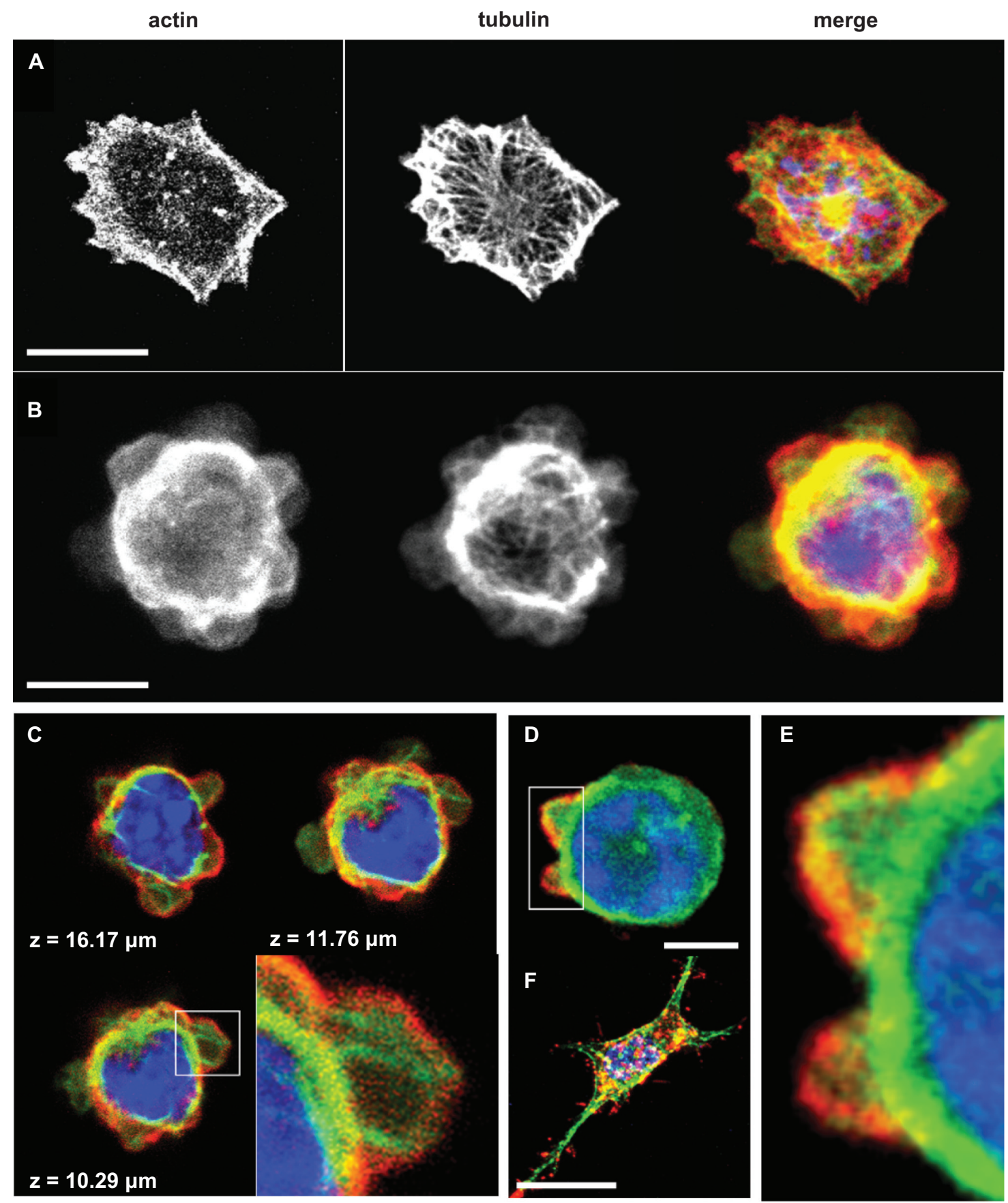

Figure $4 \mathrm{Immunofluorescence} \mathrm{imaging} \mathrm{of} \mathrm{the} \mathrm{actin} \mathrm{and} \mathrm{microtubule} \mathrm{cytoskeleton} \mathrm{in} \mathrm{flattened} \mathrm{and} \mathrm{round} \mathrm{EI4tg2a} \mathrm{mESC.} \mathrm{(Unless} \mathrm{indicated,} \mathrm{images} \mathrm{are} \mathrm{z-projections} \mathrm{of} \mathrm{each}$ confocal slice.) A) In flattened cells (scale bar $=20 \mu \mathrm{m}$ ), we observed a complete lack of F-actin and a well-defined tubulin network. B) In round cells (scale bar $=10 \mu \mathrm{m}$ ), actin is found throughout the cell and around the cell edges. The microtubule network is intact and forms a cortical mesh around the cell body. C) Single confocal planes of the cells in B, where actin is clearly observable around the bleb edges and intact microtubules are observed extending from the inner cell into several blebs (zoomed image from the plane at $z=10.29 \mu \mathrm{m}$ ). The nucleus also occupies a large volume of the cell. D) Imaging of round cells typically reveals $p E R M$ (red) localized in surfaces of spontaneously formed blebs (scale bar $=2 \mu \mathrm{m}$ ). A zoom on the blebs (D) shows that actin (green) and the pERM are localized together as expected. F) In contrast, flattened cells have $\mathrm{PERM}$ proteins evenly distributed around the cell (scale bar $=20 \mu \mathrm{m})$.

throughout the cell (Figure 4A), and in round cells, the intact tubulin network was typically found throughout the cell surrounded by an actin cortical mesh (Figure 4B). Both round and flattened cellular morphological subpopulations appeared to have a similar distribution of filamentous tubulin in their cytoskeletons. Interestingly, microtubules were also found to extend from the inner cell to the inside of spontaneously formed blebs (Figure 4C). This may account for the stability of these pre-existing blebs during application of force via AFM and the longer time-scale growth and retraction 
dynamics of spontaneously formed blebs compared to miBlebs. ${ }^{21}$ Blebbing can be observed during apoptosis, but apoptotic blebs do not have F-actin around the edge of the bleb. ${ }^{22,23}$ F-actin was observed at the leading edge of the bleb, supporting our earlier results showing that blebs were not apoptotic.

When we examined the presence and distribution of pERM proteins, we found very little activated pERM in round cells compared to flattened cells (Figure 4D, F). However, pERM proteins were present sporadically under the cell membrane and were typically localized along the edges of spontaneously formed blebs (Figure 4E). The presence of pERM in the blebs is significant as it indicates that the pERM-positive blebs were undergoing retraction. Therefore, the activation and recruitment of ERM proteins to link the cytoskeleton to the plasma membrane is as expected. ${ }^{14}$ In contrast, flattened cells possessed pERM proteins uniformly across the cell body. This difference in the distribution of pERM activity is likely one of the governing factors which determines the ability of mESC to form miBlebs. The increased pERM throughout the flattened cells acts to cross-link the plasma membrane to the cytoskeleton and provides a strong mechanical link which prevents the formation of spontaneous blebs and miBlebs. Furthermore, as we have already established that flattened cells have begun to differentiate, we suggest that pERM activation may be an early indicator of differentiation. ERM proteins and the cytoskeleton are crucial for a cell to establish a morphological phenotype. ${ }^{5}$ Furthermore, pERM in conjunction with integrin dynamics can dictate a cell's ability to sense its mechanical environment. ${ }^{2}$ The results presented here are consistent with previous work that has established the relationship between cell morphology, matrix mechanics, and the cytoskeleton with cell fate..$^{2-5}$

\section{Discussion}

Blebs form spontaneously, primarily as a result of internal intracellular forces and pressure. ${ }^{15}$ Spontaneous blebbing may have a number of different functions, including plasma membrane formation, the generation of cytoskeletal proteins during cell division, motility, growth, and expansion, and also demonstrates individual cell plasticity. ${ }^{14-16}$ We have shown that spontaneous blebbing occurs in a subpopulation of single round $\mathrm{mESC}$ and not in a subpopulation of flattened cells. It has been previously shown that seeding mESC at a low seeding density induces differentiation in the presence of LIF and speeds differentiation in the absence of LIF. ${ }^{24,25} \mathrm{LIF}$, required by mESC for the maintenance of pluripotency, can exist as a soluble factor in the medium or bound to the ECM. ${ }^{24,26}$ It has been suggested that spontaneous differentiation at low cell densities could be caused by insufficient ECM production resulting in a deficiency of ECM-associated LIF. ${ }^{27}$ Whatever the mechanism, our findings show that a subpopulation exhibiting morphological traits of differentiation can be observed just $14-18 \mathrm{~h}$ after plating at a low cell density.

Although round undifferentiated mESC display spontaneous blebbing, we also observed the formation of miBlebs upon application of 5 and $10 \mathrm{nN}$ of force. Typically, upon application of force, 1-3 miBlebs formed within $10 \mathrm{sec}$, and they were often at least twice as large as spontaneously formed blebs. After removal of external force, we observed bleb retraction after $\sim 30 \mathrm{sec}$. During this time, it is likely that the machinery for bleb retraction (actin, myosin II, ERM proteins, etc.) is recruited to the miBleb and the kinetics observed here are consistent with previously reported dynamics. ${ }^{14}$ miBlebs formed in 1-2 sec expanded to an equilibrium in size within 10-30 sec and after the removal of force, retracted within another $30 \mathrm{sec}$. A previous study found blebs forming and retracting within $\sim 30 \mathrm{sec} .{ }^{14}$ Importantly, in flattened cells undergoing early differentiation, we observed no miBleb formation.

In vivo, ESC are located in the inner cell mass of the developing embryo. ${ }^{28}$ Although no direct measurements of the mechanical properties of the inner cell mass have been made, unfertilized Xenopus eggs and the blastula wall of sea urchins were shown to be soft when compared to the majority of adult tissues. ${ }^{2,29,30}$ Studies on ESC are now revealing that differentiation leads to an increase in mechanical strength. Magnetic twisting cytometry has been used to show that mESCs are less mechanically strong than differentiated cells. ${ }^{31}$ Differentiated cells exhibited a stronger mechanical link between the cell membrane and the cytoskeleton, and stress-induced spreading occurred in undifferentiated mESC but not in differentiating cells. In a separate study, AFM was used to show that undifferentiated ESCs are more flexible than differentiating mESCs. ${ }^{32}$ Our finding that miBlebs are only induced in pluripotent ESCs supports the previous findings that ESCs are less mechanically stable than differentiating ESCs. Although the functional consequence of cell stiffening during differentiation remains unclear, a number of studies have shown that extracellular forces can affect early development. For example, it has been found that the biomechanical forces generated by fluid flow are crucial for early hematopoiesis. ${ }^{10}$ In another study, Drosophila embryos expressed the TWIST gene, which is important during the development of the digestive system, after the embryos were compressed and exposed to extracellular forces. ${ }^{33}$ 
ERM proteins are cross-linking proteins between the cytoplasm membrane-associated proteins and the actin cytoskeleton and are known to be involved in the formation of microvilli, cell adhesion sites, ruffling membranes, cleaving furrows, regulation of the actin cytoskeleton, cell shape adhesion and motility, and cellular junctions. ${ }^{34-37}$ ERM proteins are found in the cytosol of cells and are believed to be either in an active (insoluble) state or in an inactive (soluble) state. ${ }^{34}$ ERM binding at the four-point-one ERM domain by neutral endopeptidase cytoplasmic tail is involved in actin cytoskeleton organization and cell proliferation. . $^{34,36-38}$ We observed a uniform, widespread distribution of ERM proteins in flattened cells suggesting that early differentiation is associated with increased pERM activity. The more evenly distributed $\mathrm{pERM}$ proteins provide further links between the cytoskeleton and the plasma membrane, which clearly results in greater mechanical stability. However, it is unclear whether ERM phosphorylation is in response to differentiation or an integral element of the pluripotency machinery. pERM activation could be an important early marker of mESC differentiation as it is clearly linked with well-recognized morphological traits of differentiation and precedes complete downregulation of traditional pluripotency markers such as OCT4 and Nanog.

Round undifferentiated ESCs are clearly sensitive to mechanical forces due to weak membrane-cytoskeleton linkages, and this sensitivity is lost very early in the differentiation pathway. This appears to be a result of the development of a morphological phenotype and pERM activation and provides valuable insight into our understanding of the interplay of the mechanical environment and the process of differentiation. Clearly, mechanosensitivity of ESC at the earliest stages of differentiation has an important, yet still poorly understood, role in differentiation and development. These findings have important implications for the control of forces during the production of robust, reproducible regenerative medicines from ESC and implicate pERM activation as a valuable marker of early differentiation.

\section{Acknowledgments}

ALH thanks EPSRC, UK and The UK Stem Cell Bank, NIBSC, UK. DH acknowledges the Technology Strategy Board. AEP acknowledges financial support from the IRC in Nanotechnology (EPSRC, UK) and the Dr Mortimer and Mrs Theresa Sackler Trust. AEP gratefully acknowledges the Canada Research Chairs program for partial support of this work.

\section{Disclosure}

The authors report no conflicts of interest in this work.

\section{References}

1. Thomson H. Bioprocessing of embryonic stem cells for drug discovery. Trends Biotechnol. 2007;25(5):224-230.

2. Engler AJ, Sen S, Sweeney HL, Discher DE. Matrix elasticity directs stem cell lineage specification. Cell. 2006;126(4):677-689.

3. Even-Ram S, Artym V, Yamada KM. Matrix control of stem cell fate Cell. 2006;126(4):645-647.

4. Huang S, Ingber DE. Cell tension, matrix mechanics and cancer development. Cancer Cell. 2005;8(3):175-176.

5. Kunda P, Pelling AE, Liu T, Baum B. Moesin controls cortical rigidity, cell rounding, and spindle morphogenesis during mitosis. Curr Biol. 2008;18(2):91-101.

6. McBeath R, Pirone DM, Nelson CM, Bhadriraju K, Chen CS. Cell shape, cytoskeletal tension, and RhoA regulate stem cell lineage commitment. Dev Cell. 2004;6(4):483-495.

7. Saha S, Ji L, de Pablo JJ, Palecek SP. Inhibition of human embryonic stem cell differentiation by mechanical strain. J Cell Physiol. 2006; 206(1):126-137.

8. Saha S, Ji L, de Pablo JJ, Palecek SP. TGFbeta/Activin/Nodal pathway in inhibition of human embryonic stem cell differentiation by mechanical strain. Biophys J. 2008;94(10):4123-4133.

9. Veraitch FS, Scott R, Wong JW, Lye GJ, Mason C. The impact of manual processing on the expansion and directed differentiation of embryonic stem cells. Biotechnol Bioeng. 2008;99(5):1216-1229.

10. Adamo L, Naveiras O, Wenzel PL, et al. Biomechanical forces promote embryonic haematopoiesis. Nature. 2009;459(7250): 1131-1135.

11. Shimizu N, Yamamoto K, Obi S, et al. Cyclic strain induces mouse embryonic stem cell differentiation into vascular smooth muscle cells by activating PDGF receptor beta. J Appl Physiol. 2008;104(3): 766-772.

12. Binnig G, Quate CF, Gerber C. Atomic force microscope. Phys Rev Lett. 1986;56(9):930-933.

13. Haupt BJ, Pelling AE, Horton MA. Integrated confocal and scanning probe microscopy for biomedical research. Scientific World Journal. 2006;6:1609-1618.

14. Charras GT, Hu CK, Coughlin M, MitchisonTJ. Reassembly of contractile actin cortex in cell blebs. J Cell Biol. 2006;175(3):477-490.

15. Charras G, Paluch E. Blebs lead the way: how to migrate without lamellipodia. Nat Rev Mol Cell Biol. 2008;9(9):730-736.

16. Charras GT. A short history of blebbing. J Microsc. 2008;231(3): 466-478.

17. Ying QL, Nichols J, Chambers I, Smith A. BMP induction of Id proteins suppresses differentiation and sustains embryonic stem cell self-renewal in collaboration with STAT3. Cell. 2003;115(3):281-292.

18. Pelling AE, Veraitch FS, Chu CP, Mason C, Horton MA. Mechanical dynamics of single cells during early apoptosis. Cell Motil Cytoskeleton. 2009;66(7):409-422.

19. Heng BC, Liu H, Cao T. Can the high nuclear to cytoplasmic ratio of human embryonic stem cells make them more vulnerable to physical stress encountered with bulk-passage protocols? Med Hypotheses. 2005; 64(6):1242-1243.

20. Smith AG, Heath JK, Donaldson DD, et al. Inhibition of pluripotential embryonic stem cell differentiation by purified polypeptides. Nature. 1988;336(6200):688-690.

21. Tournaviti S, Hannemann S, Terjung S, et al. SH4-domain-induced plasma membrane dynamization promotes bleb-associated cell motility. J Cell Sci. 2007;120(Pt 21):3820-3829.

22. Endresen PC, Fandrem J, Eide TJ, Aarbakke J. Morphological modifications of apoptosis in HL-60 cells: effects of homocysteine and cytochalasins on apoptosis initiated by 3-deazaadenosine. Virchows Arch. 1995;426(3):257-266. 
23. Laster SM, Mackenzie JM Jr. Bleb formation and F-actin distribution during mitosis and tumor necrosis factor-induced apoptosis. Microsc Res Tech. 1996;34(3):272-280.

24. Rathjen PD, Toth S, Willis A, Heath JK, Smith AG. Differentiation inhibiting activity is produced in matrix-associated and diffusible forms that are generated by alternate promoter usage. Cell. 1990; 62(6):1105-1114.

25. Zandstra PW, Le HV, Daley GQ, Griffith LG, Lauffenburger DA. Leukemia inhibitory factor (LIF) concentration modulates embryonic stem cell self-renewal and differentiation independently of proliferation. Biotechnol Bioeng. 2000;69(6):607-617.

26. Mereau A, Grey L, Piquet-Pellorce C, Heath JK. Characterization of a binding protein for leukemia inhibitory factor localized in extracellular matrix. J Cell Biol. 1993;122(3):713-719.

27. Templeton NS, Roberts DD, Safer B. Efficient gene targeting in mouse embryonic stem cells. Gene Ther. 1997;4(7):700-709.

28. Thomson JA, Itskovitz-Eldor J, Shapiro SS, et al. Embryonic stem cell lines derived from human blastocysts. Science. 1998;282(5391): 1145-1147.

29. Valentine MT, Perlman ZE, Mitchison TJ, Weitz DA. Mechanical properties of Xenopus egg cytoplasmic extracts. Biophys J. 2005;88(1): 680-689.

30. Davidson LA, Oster GF, Keller RE, Koehl MA. Measurements of mechanical properties of the blastula wall reveal which hypothesized mechanisms of primary invagination are physically plausible in the sea urchin Strongylocentrotus purpuratus. Dev Biol. 1999;209(2): $221-238$.
31. Chowdhury F, Na S, Li D, et al. Material properties of the cell dictate stress-induced spreading and differentiation in embryonic stem cells. Nat Mater. 2010;9(1):82-88.

32. Pillarisetti A, Ladjal H, Ferreira A, Keefer C, Desai JP. Mechanical characterization of mouse embryonic stem cells. Conf Proc IEEE Eng Med Biol Soc. 2009;1:1176-1179.

33. Farge E. Mechanical induction of twist in the Drosophila foregut/ stomodeal primordium. Curr Biol. 2003;13(16):1365-1377.

34. Tsukita S, Yonemura S. Cortical actin organization: lessons from ERM (ezrin/radixin/moesin) proteins. J Biol Chem. 1999;274(49): 34507-34510.

35. Gautreau A, Louvard D, Arpin M. Morphogenic effects of ezrin require a phosphorylation-induced transition from oligomers to monomers at the plasma membrane. J Cell Biol. 2000;150(1):193-203.

36. Terawaki S, Kitano K, Hakoshima T. Structural basis for type II membrane protein binding by ERM proteins revealed by the radixinneutral endopeptidase 24.11 (NEP) complex. J Biol Chem. 2007; 282(27):19854-19862.

37. Hughes SC, Fehon RG. Understanding ERM proteins - the awesome power of genetics finally brought to bear. Curr Opin Cell Biol. 2007; 19(1):51-56.

38. Matsui T, Maeda M, Doi Y, et al. Rho-kinase phosphorylates COOHterminal threonines of ezrin/radixin/moesin (ERM) proteins and regulates their head-to-tail association. J Cell Biol. 1998;140(3):647-657. 


\section{Supplementary experimental procedures}

\section{Immunofluorescent staining and imaging}

All cells were fixed with $3.5 \%$ paraformaldehyde (Sigma), $2 \%$ sucrose (Sigma) at $37^{\circ} \mathrm{C}$ for $10 \mathrm{~min}$. Cells were washed with warm PBS (Sigma) and then permeabilized with $0.1 \%$ Triton (Sigma) at $37^{\circ} \mathrm{C}$ for 3 min and washed again with PBS. Finally, cells were blocked in wash buffer (5\% FCS in PBS for $20 \mathrm{~min}$ ). For cytoskeleton and pERM staining, cells were plated on gelatin-coated 50-mm glass-bottomed dishes (World Precision Instruments). Actin was stained with Phalloidin conjugated to Alexa Fluor 546 or 488 (Invitrogen) for $20 \mathrm{~min}$ at room temperature followed by incubation in wash buffer for $15 \mathrm{~min}$. Cells were then rinsed in ice-cold PBS. All subsequent steps were carried out on ice. Cells were incubated with mouse monoclonal $\alpha$-tubulin (Sigma) for $15 \mathrm{~min}$ or rabbit polyclonal phosphor-ERM (pERM) antibodies overnight in the fridge (Cell Signalling Technologies, Danvers, MA), a kind gift of Dr. Buzz Baum, MRC Laboratory for Molecular Cell Biology, followed by incubation with wash buffer for another $15 \mathrm{~min}$. Rabbit anti-mouse or goat anti-rabbit secondary antibodies conjugated to Alexa Fluor 488 and 647 (Invitrogen), respectively, were applied to the cells followed by wash buffer, each for $15 \mathrm{~min}$. Finally, the cells were rinsed in ice-cold PBS and nuclei were stained with DAPI (Invitrogen) for $5 \mathrm{~min}$. Cells were washed 3 times with PBS and mounted in DABCO Antifade (Sigma) and imaged.

To stain for Nanog, cells were fixed and permeabilized as above before being incubated with rabbit polyclonal Nanog (Abcam, Cambridge, UK) for $1 \mathrm{~h}$ at $37^{\circ} \mathrm{C}$. Cells were then washed 3 times with PBS and incubated with anti-rabbit secondary antibodies conjugated to Alexa Fluor 647 (Abcam) and left on ice for $1 \mathrm{~h}$ under foil. The cells were washed with PBS and stained with DAPI as above. Finally, cells were rinsed and imaged in PBS. To stain for OCT4, cells were fixed and permeabilized as above and mouse monoclonal OCT4 (Santa Cruz Biotechnology, Santa Cruz, CA) was added to the cells at a dilution of 1:200 in PBS and left for $1 \mathrm{~h}$ at $37^{\circ} \mathrm{C}$. The cells were then washed 3 times with PBS and incubated with anti-mouse Alexa Fluor 555 at a dilution of 1:500 and left on ice for just under an hour. The cells were then washed with PBS and imaged. OCT4-GFP expression in OCT4-GiP cells was directly imaged in the growth medium without any treatment. During image acquisition, the exposure time and gain settings were kept constant for each assay.

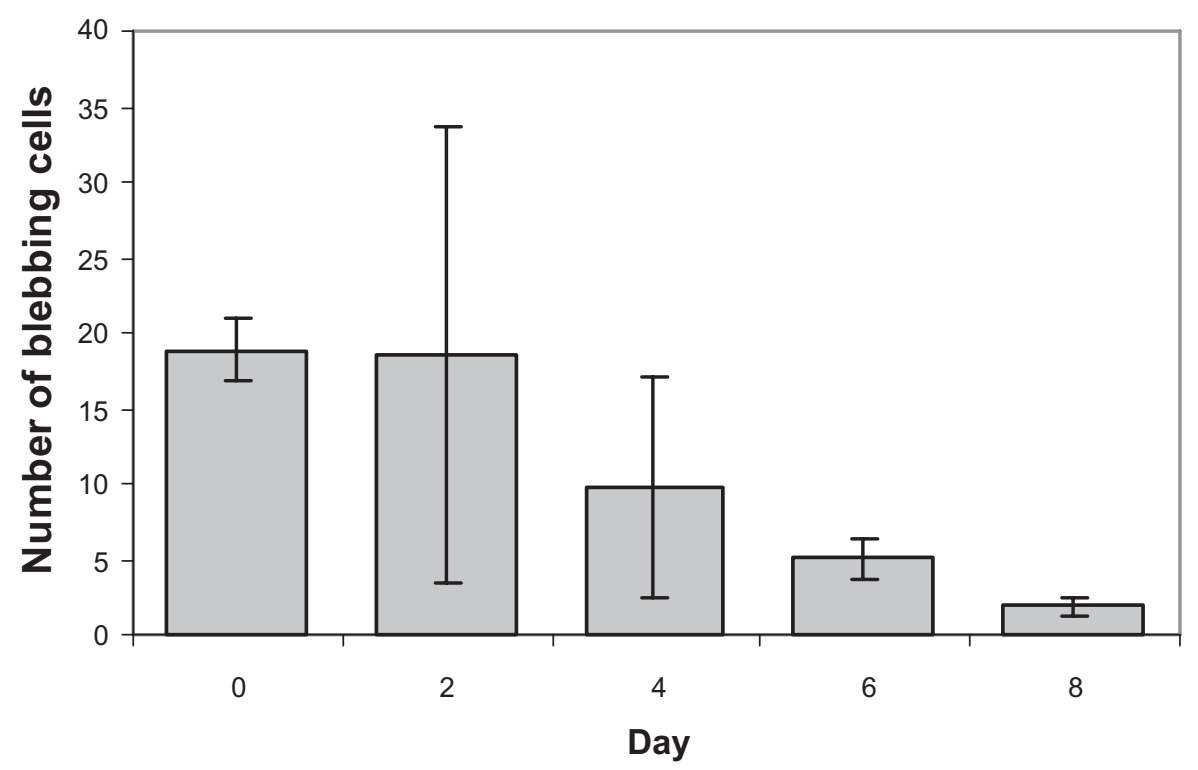

Figure SI The effect of LIF on the ability of single cells to form spontaneous blebs was analyzed by light microscopy. For the duration of the experiment, the cells were passaged every $48 \mathrm{~h}$ and analyzed the following day. Single cells were plated onto gelatinized 60 -mm Iwaki Petri dishes and left to attach overnight before analysis. At each time point, $\sim 150$ single cells were monitored manually in triplicate cultures $(n=3)$. The percentage of single cells exhibiting spontaneous blebs was calculated as the number of blebbing cells divided by the total number of cells counted. As cells begin to differentiate, the ability to spontaneously bleb significantly deceases (day 0 to day $8, P \leq 0.05$ ). 


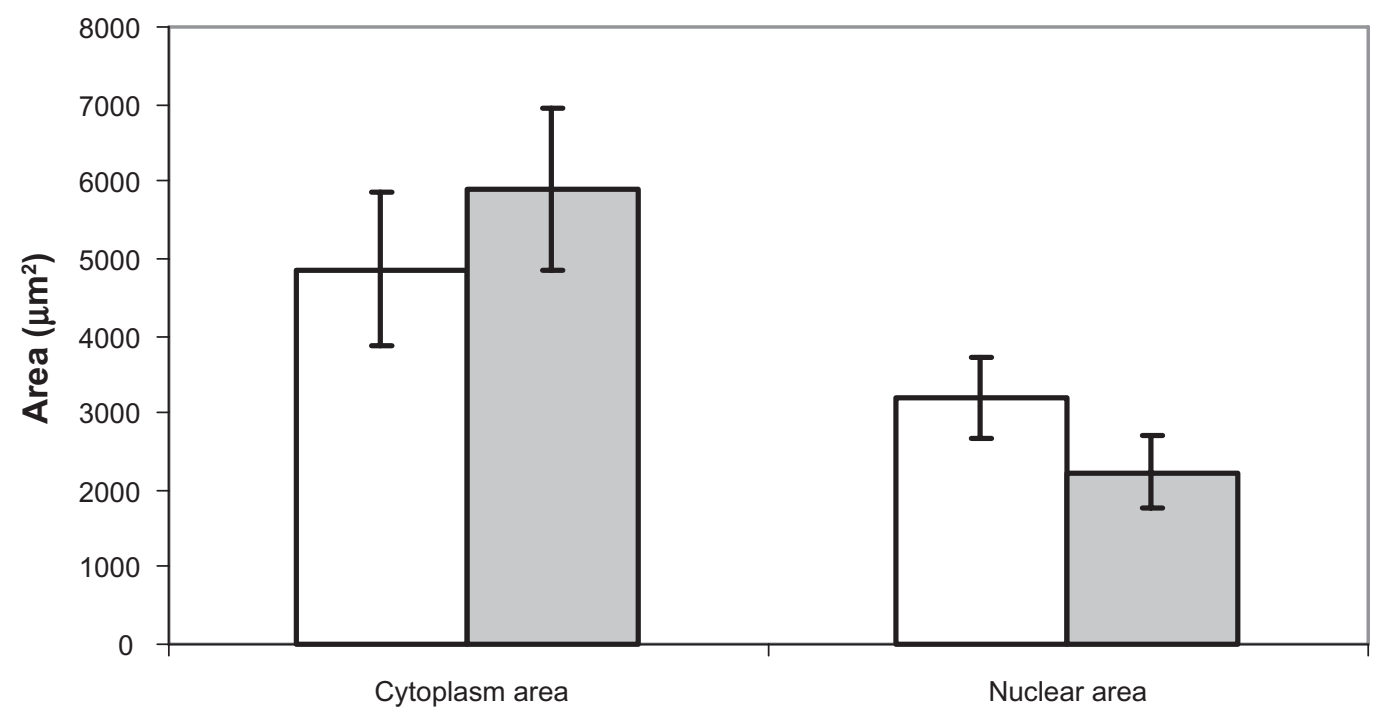

Figure S2 Analysis of cytoplasm and nuclear areas in round (no fill) and flat (gray fill) cells.
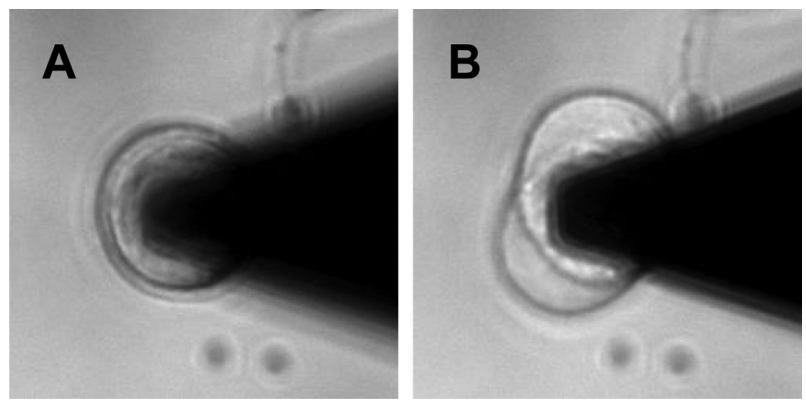

Figure S3 A) Outline of the cell before the application of force. B) Application of $5 \mathrm{nN}$ of force to a cell with round morphology leading to the formation of a miBleb.

\section{Publish your work in this journal}

Cell Health and Cytoskeleton is an international, peer-reviewed open access journal focusing on all aspects of cell structure and function contributing to normal physiology and cell health and exploring the pathogenesis of cell dysfunction leading to adverse conditions and disease in the organism. The journal welcomes papers covering original research, basic science, reviews and evaluations, guidelines, expert opinion and commentary, case reports and extended reports. The manuscript management system is completely online and includes a very quick and fair peer-review system, which is all easy to use. Visit http://www.dovepress. com/testimonials.php to read real quotes from published authors. 